\title{
Human-machine cooperation: a solution for life-critical Systems?
}

\author{
Patrick Millot ${ }^{\mathrm{a}, \mathrm{b}}$ and Guy A. Boy ${ }^{\mathrm{b} *}$ \\ ${ }^{a}$ LAMIH CNRS Université de Valenciennes, Le Mont Houy 59313 Valenciennes Cedex 9, France \\ ${ }^{b}$ HCDi, Florida Institute of Technology, 150 West University Blvd, Melbourne, FL 32901, U.S.A.
}

\begin{abstract}
Decision-making plays an important role in life-critical systems. It entails cognitive functions such as monitoring, as well as fault prevention and recovery. Three kinds of objectives are typically considered: safety, efficiency and comfort. People involved in the control and management of such systems provide two kinds of contributions: positive with their unique involvement and capacity to deal with the unexpected; and negative with their ability to make errors. In the negative view, people are the problem and need to be supervised by regulatory systems in the form of operational constraints or by design. In the positive view, people are the solution and lead the game; they are decision-makers. The former view also deals with error resistance, and the latter with error tolerance, which, for example, enables cooperation between people and decision support systems (DSS). In the real life, both views should be considered with respect to appropriate situational factors, such as time constraints and very dangerous environments. This is known as function allocation between people and systems. This paper presents a possibility to reconcile both approaches into a joint human-machine organization, where the main dimensioning factors are safety and complexity. A framework for cooperative and fault tolerant systems is proposed, and illustrated by an example in Air Traffic Control.
\end{abstract}

Keywords: life critical systems, human error, cooperative decision support system, fault tolerant system, air traffic management.

\section{Introduction}

In the human-machine systems field of research, the term "machine" refers not only to computers, but also to diverse complex dynamic systems, such as industrial processes, transportation systems and communication networks. Human activities are mainly oriented toward decision-making, including monitoring, fault anticipation and detection, diagnosis and prognosis, as well as fault prevention and recovery. Decision-making is motivated by humanmachine system performance (e.g., production quantity and quality), as well as overall system safety. Moreover decision-making should be based on "sense-making", which is more about continually revising interpretations in an ongoing stream of stimuli and activity. When people make sense of the situation, usually the decision is obvious, and in many settings they would not view themselves as having made a decision, even though they did take one action as opposed to another [36].

In most nominal operations, automation simplifies our lives. However, people need to keep their skills and knowledge to be able to master systems they manage, and must remain active in the control and supervisory loop of these systems. Indeed, automation may increase interactivity between people and machines, as well as decision-making difficulty and decision-makers and workload.

\section{The ambiguous role of people in automated systems}

Technical progress has increased machine decisional capabilities so that human-machine systems have increasingly become complex cognitive systems in which interactions between the human and artificial agents increase as the systems deal with more complex and cognitive tasks [12]. For this rea-

\footnotetext{
* Corresponding authors. Emails: patrick.millot@univ-valenciennes.fr, pmillot@fit.edu, gboy@fit.edu
} 
son, human operators must control not only the process but also the artificial agents. One major problem is linked to the respective functions allocated to the agents involved in the system, and thus to the definition of their responsibilities [7]. One of us already defined a cognitive function by three attributes, i.e. role, context of validity and a set of resources supporting the performance of the task [3]. Human operators who are responsible for complete system operations may thus have some doubts about interacting with a machine if they do not feel that they can control it completely. This very important point might be worth expanding, and is seen frequently for instance in the hospital: people won't adjust a machine because they are uncertain and do not want to screw it up.

Even when the machine has the responsibility for making the decisions and taking the actions according to the choices of the designer, the human operators can nevertheless intervene when they perceive a problem related to the system safety. This is for example the case in aeronautics with on-board anticollision systems [30].

Responsibility allocation among people and machines belongs to the general problem of sharing the authority [4,19] and thus involves human factors, such as self confidence and trust [20]. Rajaonah [25] already described confidence construction mechanisms and their impact on the relationship between people and artificial agents.

Through an evaluation of computerized decision support systems, Barr and Sharda [1] highlighted the positive and negative effects of their use by human operators. These authors pointed out, that using Decision Support Systems (1) increases human operator's understanding of the problems to be solved, (2) improves human operator's information processing performance, and (3) boosts human operator's confidence in the final decision, by allowing human operator's to focus on the strategic aspects of the problem to be solved. However, at the same time, using these computerized decision-making systems can make human operators passive, facilitating complacency, specifically because they typically ignore why the system proposes what it proposes! When human behavior depends on system's decisions, human efficiency is generally due to passivity. Moreover, people can accept an alternative from a DSS that is worse than what they would have come up with unaided [37].

Human roles can be antagonist: (1) people competence warranty system integrity and safety; and (2) people as decision-makers can make errors and then endanger the system, more specifically in case of life-critical systems.

Two types of organization currently exist, one placing the human decision-makers in the best possible environment, e.g., assisting them with DSS and allowing them to be free to take the good decision; a second, which is the opposite and considers that people can make errors and therefore must be supervised by a fault resistant system.

\section{Putting people at the right spot}

Safety is directly related to management of human-machine system complexity. It depends on three kinds of parameters contributing to complexity: technical parameters, human parameters, and parameters related to the interaction between the first two.

\subsection{HMS complexity}

Technical failures and human errors generally increase with the size and/or the complexity of the system (i.e., the number of interconnections between the controlled variables and their degree of interconnection). For example, large life-critical systems, such as power plants or transport networks, can have several thousands of interconnected variables that need to be supervised. The human supervisor must be able to understand system's behavior, in order to manage the resulting complexity, i.e., to manage the right information, at the right abstraction level, at the right moment and in time according to system dynamics. That can be obtained in classifying the tasks with respect to an abstraction hierarchy such as the following three levels: strategic, tactical and operational $[27,32,18]$. The lower or operational level is related to the process to be controlled or supervised; it is decomposed into subsystems, and their local control units. The second or tactical level coordinates local control units, including DSS. And the higher or strategic level corresponds to the human team.

As we go from the lower level up to the higher one: (1) the nature of information ranges from precise numerical data to symbolic and global information; (2) abstraction levels go from means to objectives (and conversely); (3) temporal horizons evolve from real time activities at the bottom (e.g., the subsystem control tasks) towards long-term activities (e.g., planning or strategic decision-making) at the top.

Indeed sorting tasks with respect to these three levels facilitates the definition of the nature of the human tasks and the expected task performance for each level. Human abilities are best suited to processing symbolic information, planning and anticipating decisions with respect to global objectives rather than specific means, and this on a middle- or long-term horizon. For this reason, activities situated at the bottom of the hierarchy are not well suited to human capabilities, and this can lead to human errors. Unfortunately these criteria are not always applied when designing human-machine systems. We will make a distinction between two approaches to HMS modeling: open loop and close loop. 


\subsection{Open loop human HMS modeling}

Many models have been proposed, such as Rasmussen's human behavior model [26] which includes four major functions: (1) abnormal event detection; (2) situation assessment by perceiving information for identifying (diagnosis) and/or predicting (prognosis) the process state; (3) decision-making by predicting the consequences of this state on the process goals, defining targets to be achieved and breaking these targets down into tasks and procedures; and (4) performing resulting tasks and procedures that affect the process. This model is very close to others developed in artificial intelligence [21]. Nevertheless this model has been revisited since in real world people did not proceed linearly from one step to the next, but made large jumps from one point to another, sometimes went backwards. For instance Hoc proposes a new version in which a diagnosis is the result of an iterative process between elaboration and test of hypotheses [38].

Rasmussen's model depicts three behavioral levels, which enhances its effectiveness and recalls the hierarchical view-points: (a) skill-based behavior (SBB) used by a trained human operator, who performs a task in an automatic manner when perceiving a specific signal; (b) rule-based behavior (RBB) used by an expert operator, who reuses a solution learned in the past when facing a well-known problem; and (c) knowledge-based behavior (KBB) used by a knowledgeable operator who must find a new solution facing an unknown problem. In this latter case, the operator is usually supported either by other operators or by a decision-support system in order to understand the process situation and make the right decision (therefore in a multi-agent situation). The first level is typically sub-conscious. Second and third levels are conscious and involve cognition.

\subsection{Close loop human HMS modeling}

A human operator is in a close loop when he or she tries to compensate with errors. It could be short-term compensation correcting errors on the spot, or long-term using errors to learn from them. Human errors and human reliability has been studied for the last three decades $[29,11]$. For example, an erroneous action may result from either the incorrect application of a right decision or the right application of an inappropriate decision. The erroneous decision itself can either produce a wrong solution based on a correct situation assessment or a sound solution based on an incorrect situation assessment, and so on.

Rasmussen [28] explained human error production by the need of reaching a compromise solution for three intertwined, sometimes contradictory, objectives: performance standards, imposed either by the organization or by the individual operator; system and/or operator safety; and the cognitive and physiological costs of attaining the first two objectives (e.g., workload and stress). The dimensions of these objectives are limited, and thus constrain the range of human actions, Figure 1. An action that crosses the limits may lead to loss of control, and subsequently, incidents or accidents.

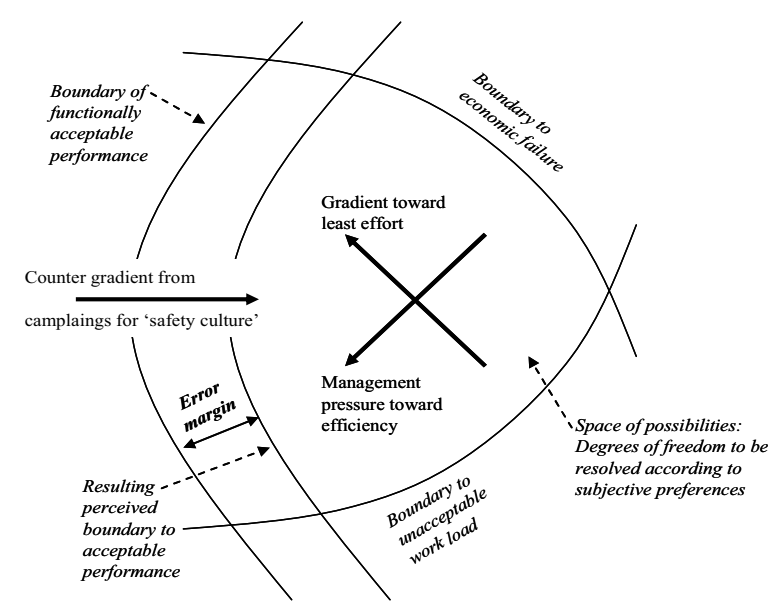

Fig. 1: Three boundary dimensions constraining the human behavior [27]

\section{Sharing tasks and functions between humans and machine}

\subsection{Degrees of Automation}

The influence of the human role and the degree of human involvement on overall human-machine system performance, regarding both production and safety, has been studied since the early 1980 s, e.g., Sheridan [32] defined the well-known degrees of automation and their consequences (see Table 1).

In a fully manual controlled system (level 1), safety depends entirely on human controller's reliability. At the opposite in a fully automated system (level 10), the human operator is not in both control and supervision loops. This degree of automation can lead to a lack of vigilance and a loss of skill of the operators involved in the supervision, which prevents them from assuming their responsibility on the system. Consequently, system safety is almost fully dependent on technical reliability.

Between these two extremes, intermediate solutions consist in inserting dedicated DSS (Decision Support Systems) and establishing supervisory control procedures that will enable authority between human operators and automated control systems.

Levels 2 to 4 correspond to a static allocation where the human operator has the control of the system but where a machine (a DSS) proposes solution(s). The human operator has the authority for controlling the system, and can either implement its own solution or choose the solution provided by the machine. Both agents interact at a tactical level to perform the task with respect to appropriate modes of human-machine cooperation. 
Table 1

Sheridan's levels of automation [34]

1 Computer offers no assistance, human do it all.

2 Computer offers a complete set of alternative actions, and

3 Narrows the selection down to a few, or

4 Suggests one, and

5 Executes that suggestion if the human approves, or

6 Allows the human a restricted time to veto before automatic execution, or

7 Executes automatically, then necessarily informs human, or

8 Informs him after execution only if he asks, or

9 Informs him after execution if it, the computers, decides to.

10 Computer decides everything and acts autonomously, ignoring the human.

At levels 5 and 6, strategic authority allocation is integrated in task performance.

Levels 7 to 9 correspond to a static allocation where the machine has the authority for implementing the solutions. These levels differ in the kind of feedback provided to the human operator.

This ten degree scale mixes tactical and strategic aspects of task performance and function allocation. Intermediate levels of automation could be added to cope with specific contexts. For example, in case of emergency, Inagaki [13] defines the level 6.5 where "the computer executes automatically upon telling the human what it is going to do". At this level, the machine performs the actions on the system for safety reasons, but informs human operator in order to reduce the automation surprise and to maintain situation awareness.

Parasuraman et al. [23] proposed to extend this approach through a simplified version of the Rasmussen's model in four steps: information elaboration, identification, decision-making and implementation of the decision. For each step the scale of automation is applied, allowing a better representation of the interactions between the agents, an allocation of subtasks (static and dynamic), and the sharing of authority between the agents for task performance and function allocation.

\subsection{Task-sharing criteria}

We make the distinction between task and activity: the task is a prescription, and the activity is what is effectively performed. Since we claim that a cognitive function transforms a task into an activity, i.e., a function performs a task to produce an activity [3] during the first stage of design, while there is no system being used, task and function can be considered as the same. Therefore, task analysis is the first step in function allocation, but should not be considered as a definitive answer to the function allocation problem. In this paper, we will only emphasize task analysis.

Task analyses support human-machine tasksharing decisions according to two criteria: technical feasibility and ergonomic feasibility $[18,19]$ :

- The first criterion allows dividing the initial task set into two classes: TA tasks which are technically able to be performed automatically by the machine, and those TH tasks which cannot be performed automatically due to lack of information or due to technical or even theoretical reasons, and thus must be allocated to human operators.

- The ergonomic feasibility criterion is applied to both subsets, TA tasks and TH tasks, to evaluate the human tasks in terms of global system safety and security:

. in the subset TA tasks, some automated tasks TAh can also be performed by humans, and allocating them to the human operators can allow these operators to better supervise and understand the global system and the automated devices. The subset TAh is thus the set of the shareable tasks, i.e., which can be shared between both agents.

. in the subset TH tasks, some subtasks THa are very complex, or their complexity is increased by a very short response time. Humans performing such tasks could be aided by a Decision Support System or a Control Support System. The subset $\mathrm{THa}$ can thus be the basis of another form of human-machine cooperation in which the agents have complementary capabilities and the tasks are shared according to that.

The ergonomic feasibility criterion is based on human operator models that define the potential human resources, as well as the intrinsic limits of the operators (perceptual and/or physical) when performing the related actions. Human cognitive resources depend on the context, and human physical resources can be determined through ergonomic guidelines.

\section{Enhancing the automated system safety}

\subsection{Risk management strategy}

Technical, organizational or procedural defenses can aim at remedying faulty actions or decisions. That can be seen as if the designer would build barriers or defenses for preventing human operators from crossing these limits and remain inside the space of possibilities, Figure 1. Thus, several risk analysis methods have been proposed for detecting risky situations and providing such remedies [11, 34, $24,33]$. Usually, risk management involves three complementary steps, which must be anticipated when designing the system:

- Prevention: the first step is to prevent risky behaviors. Unexpected behaviors should be anticipated 
when designing the system, and technical, human and organizational defenses should be implemented to avoid these behaviors (e.g., norms, procedures, maintenance policies, supervisory control).

- Correction: if prevention fails, the second step allows these unexpected behaviors to be detected (e.g., alarm detection system in a power plant) and corrected (e.g., high speed train brakes).

- Containment: if the corrective action fails, an accident may occur. The third step attempts to deal with the consequences of a failed corrective action, by intervening to minimize the negative consequences of this accident (e.g., roadside emergency care).

It is very difficult to anticipate these three steps at design time because people commonly use abduction inferences more than anything else. For example, people usually act not because they have available objective 'facts' of the situation, but rather because they facilitate or forestall some good or bad future events, based on their expectations. Nevertheless, once defined, the three steps related tasks can be performed, some by the human operators and some by the machine. Several other questions must then be asked in order to allocate the tasks:

- Should (or could) these tasks be shared between the human and the machine and performed separately?

- In that case who should coordinate the task sharing?

- Should the task be performed by both the human and the machine together?

- In that case, could the task be decomposed into subtasks, and who should coordinate the decomposition and the subtask allocations?

\subsection{Human-Machine Cooperation (HMC)}

Human-Machine Cooperation (HMC) is one of the numerous complementary ways for implementing the three steps for managing risks.

The DSS assists the Human Operator in order to make her/his task easier and then to avoid s/he performs faulty actions. Therefore the DSS intervenes before the human action. The structure of the cooperative organization can be either vertical or horizontal. In the vertical structure, the DSS provides advices to the Human Operator who is the only controller of the process, Figure 2. The horizontal structure corresponds to a task sharing between the human operator and the DSS, which both can act on the process Figure 3 [17].

Experimental studies of these structures have shown the need to introduce a second class of knowhow in the DSS, called "know-how-to-cooperate" and gathering capabilities for:

- managing the interferences between the goals of each agent, human or machine (coordination),

- facilitating the other agent's goals.

This is also confirmed by Klein's paper on making automation a team player [39].

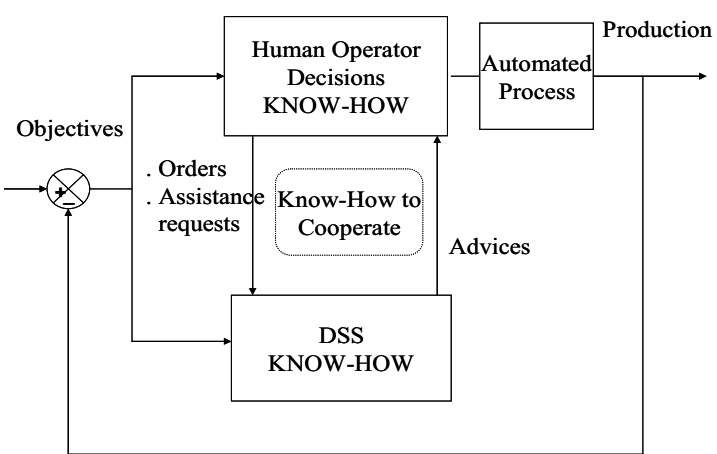

Fig. 2: Vertical Structure for human-machine cooperation

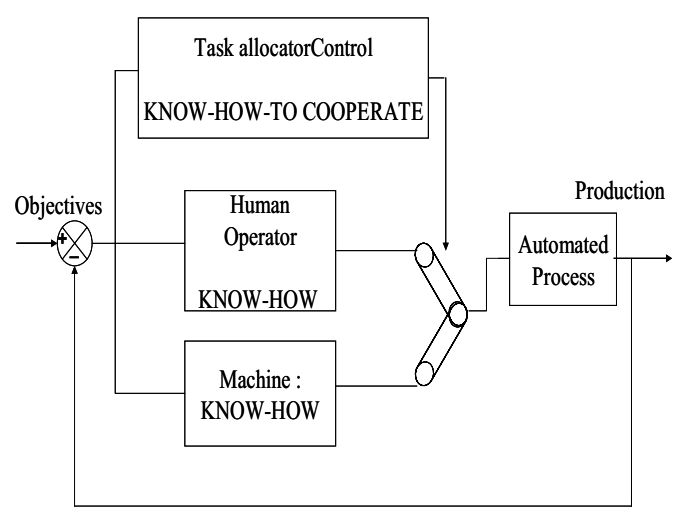

Fig. 3: Horizontal structure for human-machine cooperation

Other parameters intervene concerning the Motiva tion-to-Cooperate of the agents i.e., motivation to accomplish the task, self-confidence, trust [20], and confidence in the cooperation [25].

The know-how-to-cooperate can be implemented according to three cooperative forms [31]:

- in the augmentative form the agents have a similar know-how and they perform similar subtasks to achieve the global task,

- in the debative form the agents have a similar know-how, they process together the same task and debate before implementing actions,

- in the integrative form the agents have complementary and different know-how and perform subtasks which are complementary to achieve the task.

These three forms already exist in human-human organizations and are sometimes naturally combined. An example of the augmentative form can be observed at the bank office, when the queue before a desk is too long, a second desk is open to share the queue and to reduce the first operator's workload. An example of the debative form concerns the mutual control between flying pilot and non-flying pilot in the plane cockpit. A third example can be seen for the coordination of the different tasks needed when building a house. The innovation lies in the implementation of these forms between a human being and a machine. 


\subsection{An example in Air Traffic Control}

Generally, pure cooperative forms do not exist in the real world; most often, a combination of the three forms is encountered. This is the case in Air Traffic Control (ATC). The AMANDA (Automation and MAN-machine Delegation of Action) project has studied a new version of cooperation between Human Controllers and a new tool called STAR in the ATC context [16]. The objective of the project was to build a Common Frame of Reference, so called Common Work Space (CWS), using the support system STAR. STAR is able to take controller strategies into account in order to calculate precise solutions and then transmits the related commands to the aircraft. The Common Frame of Reference of the air traffic controllers was first identified experimentally by coding their cognitive activities.

Here, the human controllers keep the strategic authority to delegate some activities to STAR. He/she can delegate a problem to solve to STAR with some constraints represented by his/her strategies for solving a conflict, and can control the machine with the feed-backs that STAR introduces in the CWS.

The CWS was implemented on the graphic interface of the AMANDA platform [22]. The CWS plays a role similar to a blackboard, displaying the problems to be solved cooperatively. As each agent brings pieces of the solution, the CWS displays the evolution of the solution in real time.

The cooperation between STAR and the human controller can take the 3 forms, Figure 4:

- Debative for building the problems. Here, the problem is called a cluster, and is a set of conflicting aircrafts, i.e., at least two aircrafts in a duel situation (binary conflict) and other aircrafts that can interfere with this duel;

- Integrative for resolving a problem using a strategy given by the air traffic controllers. Here, the strategy is modeled as one or several "directives", and a directive can be, for example, "turn AFR365 behind AAL347";

- Augmentative for implementing the calculated solution.

The experimental evaluation showed that this cooperative organization allows the controllers to better anticipate air traffic conflicts, and then increasing the level of safety. In addition, the common work space provides a good representation of air traffic conflicts and thus appears to be a good tool for conflict resolution. Furthermore, this organization provides a better regulated workload.

As a summary, these experiments in Air Traffic Control since the early 1990 s $[35,15,16]$ have first shown an increase of the human-machine performance when the human is supported by a DSS. A second result is the need of a Common Frame Of References between both agents [22]. A third result is an important reduction (divided by 2 ) of the number of erroneous decisions and actions.

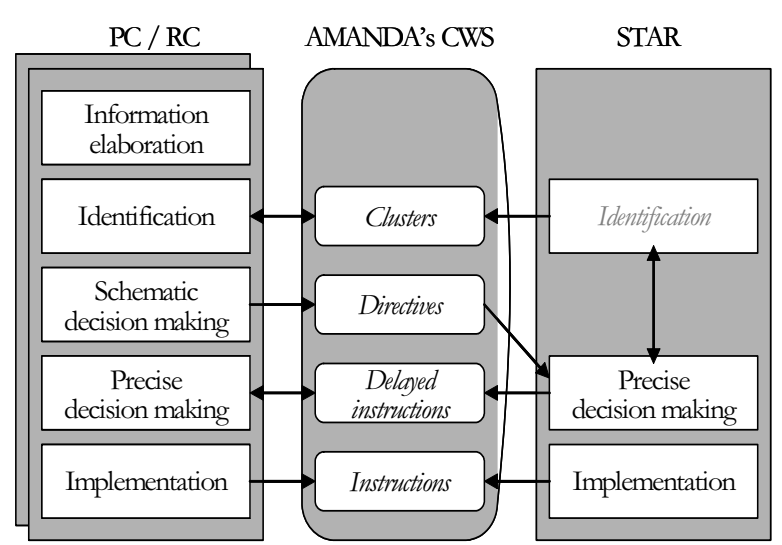

Fig. 4: AMANDA's Common Work Space Legend: $\mathrm{PC} / \mathrm{RC}=$ Planning/Radar controller

But the last and very important result is that the erroneous actions have not been cancelled and some ones still remained.

\subsection{Adding a Fault tolerant system}

As seen previously the cooperative approach does not prevent all the human errors.

We then propose to add a fault tolerant system for coping with remaining errors. Figure 5 shows a cooperative organization between a Human Operator and two systems: a decision support system (DSS) and a Fault Tolerant System (FTS). This second support system is needed for intervening after the action has been performed. It aims at evaluating the command performed on the process and at filtering erroneous commands through three possible ways: - the command can be cancelled before acting on the process under the condition that a buffer (delay, distance) has been foreseen at the design phase and set at the process input;

- if the command has already been performed on the process a safety action such as an emergency stop must be performed;

- finally, if the previous action is impossible or too late, it may be possible to act on the consequences for instance in blocking their propagation.

The main difficulties are linked to the evaluation of the command: what reference to use for deciding that the command is inadequate or not?

The present attempts for answer use a context dependent approach consisting in defining a list of prohibited commands. Variants place barriers around the process, either to avoid erroneous actions as above or to avoid unexpected process behaviors [24]. General more context free approaches could be based on more achieved models of the human errors. This needs progresses in cognitive sciences and artificial intelligence as well.

Numerous other problems emerge now, for instance linked to the need for understanding fixations 
(also called diabolic errors) [33] or trying to understand why some operators cross barriers [24].
Behind these conceptual frameworks, concrete aspects regarding implementation possibilities of such ideas must also be studied.

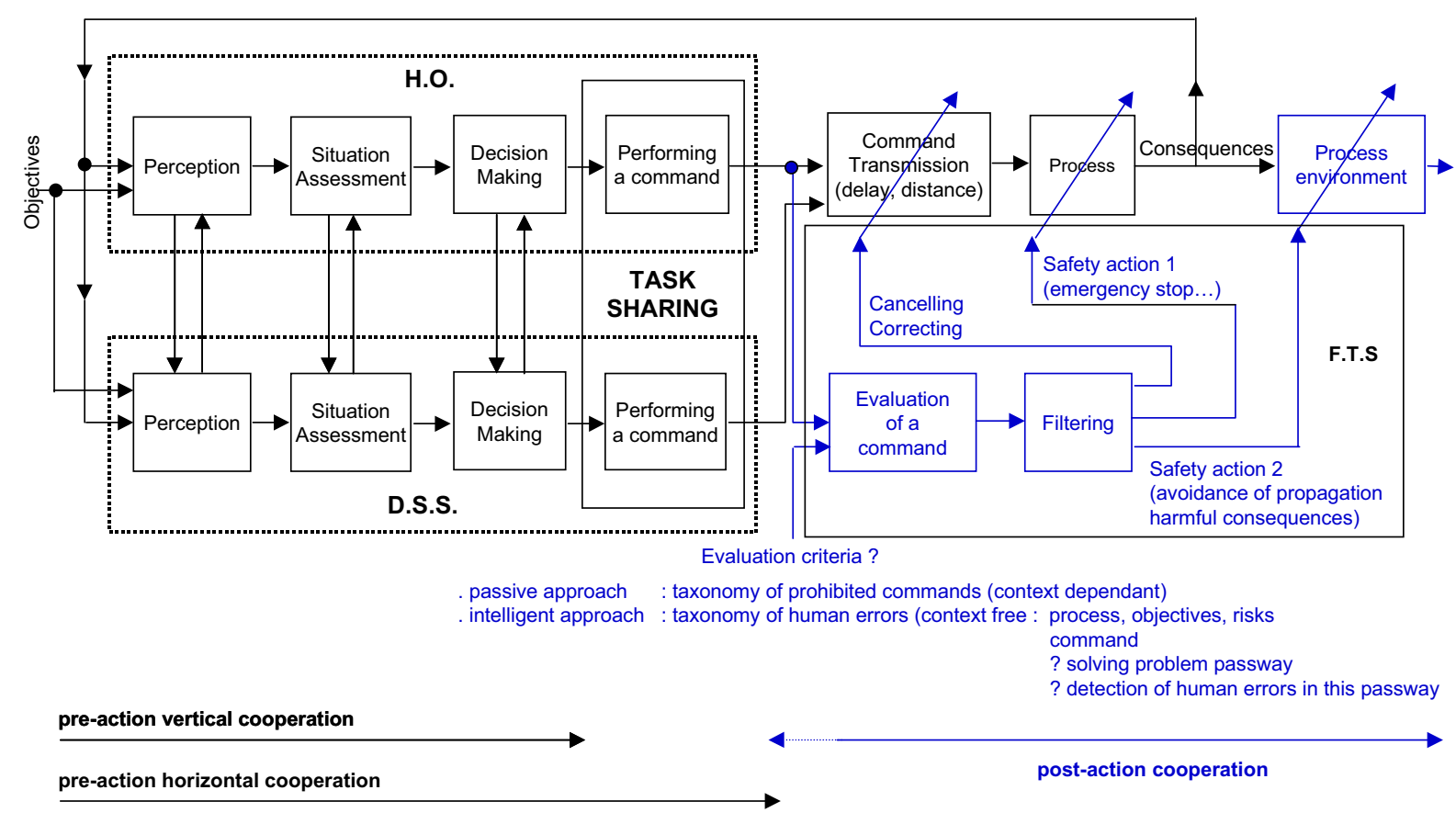

Fig. 5: Human-Machine Cooperation for safety purposes.

\section{Conclusion}

This paper has proposed a review of the objectives of human engineering and the methods used to enhance the safety of automated systems, focusing on the parameters related to human-machine cooperation -degree of automation, system complexity, and the richness and complexity of the human componentamong the different classes of parameters that influence safety. An important way of solutions consists in implementing cooperation between human operators and DSS. This paper proposes a framework for designing human-machine cooperative systems. To do so, the system needs to be able to deal with the $\mathrm{KH}$ and respective $\mathrm{KHC}$ of the different agents (human or machine). Three forms of cooperation and the notion of a common frame of reference (COFOR) have been introduced to describe the activities that make up each agent's KHC. An example of COFOR implementation through a common workspace was also presented. Finally a complementary way of thinking dealing with human errors leaded to a fault tolerant system, placed after the cooperative organization. Difficulties to implement it have been discussed.

\section{Acknowledgements}

We would like to thank Dr. Robert L Wears of the Department of Emergency Medicine, University of Florida in Jacksonville, and of the Centre for Safety and Quality Improvement, Imperial College London, for his astute comments.

\section{References}

[1] Barr S.H., Sharda R. (1997). Effectiveness of decision support systems: development or reliance effect? Decision Support Systems, Volume 21, Issue 2, October 1997, pp. 133-146.

[2] Billings CE (1997). Human-centered aircraft automation: a concept and guidelines (NASA Techn. Mem. 103885). NASA Ames Research Center.

[3] Boy G.A. (1998). Cognitive function analysis. Ablex Pub., Greenwood, CT, USA.

[4] Boy, G.A. \& Grote, G. (2011). The Authority Issue in Organizational Automation. In G.Boy (Ed) Handbook for HumanMachine Interaction. Ashgate Publishing Ltd, Wey Court East, Union Road, Farnham, Surrey, GU9 7PT, England ,2011

[5] Carlier, X., Hoc, J.M. (1999). Role of a common frame of reference in cognitive cooperation: sharing tasks in AirTraffic-Control. CSAPC'99. Villeneuve d'Ascq, France, September.

[6] Decortis, F., Pavard, B. (1994) Communication et coopération : de la théorie des actes de langage à l'approche ethnométhodologique. In Pavard B. (Ed.), Systèmes Coopératifs de la modélisation à la conception. Toulouse, France, Octarès.

[7] Debernard, S., Hoc, J-M., (2001). Designing Dynamic Human-Machine Task Allocation in Air Traffic Control: Lessons Drawn From a Multidisciplinary Collaboration. In M.J. Smith, G. Salvendy, D. Harris, R. Koubek (Ed.), Usability evaluation and Interface design: Cognitive Engineering, Intelligent Agents and Virtual Reality, volume 1. London: Lawrence Erlbaum Associate Publishers, pp. 1440-1444.

[8] Fadier E.,Actigny B. (1994). Etat de l'art dans le domaine de la fiabilité humaine. Ouvrage collectif sous la direction de E. Fadier, Octarès, Paris.

[9] Grote G., Weik S., Wäfler T., Zölch M., (1995). Criteria for the complementarity allocation of functions in automated work systems and their use in simultaneous engineering 
projects. Int. Journal. of Industrial Ergonomics, Vol. 16, № 4 6, October, pp. 367-382.

[10] Grislin, E., Millot, P. (1999). Specifying artificial cooperative agents through a synthesis of several models of cooperation. Seventh European Conference on Cognitive Science Approaches to Process Control, Villeneuve d'Ascq, 21-24 Septembre 1999, France, pp 73-78.

[11] Hollnagel, E. (1999). Cognitive Reliability and Errors Analysis Method: CREAM. Elsevier, Amsterdam.

[12] Hollnagel E. (2003). Prolegomenon To Cognitive Task Design Handbook of Cognitive Task Design, Erik Hollnagel (Ed.), Lawrence Erlbaum Associates, Publishers, London, 2003, pp. 3-15.

[13] Inagaki T.(2006). Design of human-machine interactions in light of domain-dependence of human-centered automation. Cognition, Technology and Work. Volume 8, Issue 3, pp. 161 - 167, ISSN:1435-5558.

[14] Millot, P. (1998). Concepts and limits for Human-Machine Cooperation, IEEE SMC CESA'98 Conference, Hammamet, Tunisia, April.

[15] Millot P., Lemoine, (1998) An attempt for generic concept toward human machine cooperation. IEEE SMC'98, San Diego, US.

[16] Millot P., Debernard S. (2007). An Attempt for conceptual framework for Human-Machine Cooperation. IFAC/ IFIP/IFORS/IEA Conference Analysis Design and Evaluation of Human-machine Systems Seoul Korea, September.

[17] Millot P., Hoc J.M. (1997). Human-Machine Cooperation: Metaphor or possible reality? European Conference on Cognitive Sciences, ECCS'97, Manchester UK, April.

[18] Millot P. (1999). Systèmes Homme-Machine et Automatique. Journées Doctorales d'Automatique JDA'99, Conférence Plénière, Nancy, septembre

[19] Millot P., Debernard S., Vanderhaegen F. (2011). Authority and cooperation between humans and machines. In G.Boy (Ed) Handbook for Human-Machine Interaction. Ashgate Publishing Ltd, Wey Court East, Union Road, Farnham, Surrey, GU9 7PT, England ,2011

[20] Moray N., Lee, Muir, (1995). Trust and Human Intervention in automated Systems". in Hoc, Cacciabue, Hollnagel (Eds) : Expertise and Technology cognition and Human Computer Interaction. Lawrence Erlbaum Publ.

[21] Newell, A. (1990). Unified Theories of Cognition. Harvard University Press, Cambridge, Massachusetts.

[22] Pacaux-Lemoine, M.-P., Debernard, S., (2002). Common work space for human-machine cooperation in air traffic control. Control Engineering Practice. 10 (2002) 571-576.

[23] Parasuraman R., Sheridan T.B., Wickens C.D. (2000). A Model for Types and Levels of Human Interaction with Automation. IEEE Transactions on System, Man and Cybernetics, Vol. 30, N 3. (May 2000), pp. 286-297.

[24]Polet, P., Vanderhaegen, F., Wieringa P.A. (2002). Theory of Safety-related violations of a System Barriers", Cognition Technology and Work, 4 (2002) 171-179.
[25] Rajaonah B., Tricot N., Anceaux F., Millot P. (2008). Role of intervening variables in driver-ACC cooperation, International Journal of Human Computer Studies 66 (2008) 185-197

[26] Rasmussen, J. (1983). Skills, Rules and Knowledge: signals, signs and symbols and other distinctions in human performance models: IEEE Transaction on Systems, Man and Cybernetics $\mathrm{SMC} \mathrm{n}{ }^{\circ} 3$

[27] Rasmussen J. (1991). Modelling distributed decision making. in Rasmussen J., Brehmer B., and Leplat J. (Eds), Distributed decision-making: cognitive models for cooperative work pp111-142, John Willey and Sons, Chichester UK.

[28] Rasmussen J. (1997). Risk management in a dynamic society: a modelling problem. Safety Sciences, 27, 2/3, (1997) 183-213.

[29] Reason J. (1993). Human error. Cambridge University Press (1990) (Version française traduite par J.M. Hoc, L'erreur humaine, PUF.

[30]Rome F., Cabon P., Favresse A., Mollard R., Figarol S., Hasquenoph B. (2006). Human Factors Issues of TCAS: a Simulation Study. International Conference on Human-Computer Interaction in Aeronautics (HCI - Aero 2006), Seattle, Washington, 20-22 september, 2006.

[31] Schmidt, K. (1991). Cooperative work: a conceptual framework. In J. Rasmussen, B. Brehmer, \& J. Leplat (Eds.), Distributed decision-making: cognitive models for cooperative work. Chichester, UK : John Willey and Sons. pp. 75-110.

[32] Sheridan, T.B. (1992). Telerobotics, Automation, and Human Supervisory Control. MIT Press, Cambridge, MA.

[33] Van der Vlugt M., Wieringa P.A, (2003). Searching for ways to recover from fixation. Cognitive Science Approach for Process Control CSAPC'03, Amsterdam, September.

[34] Vanderhaegen, F. (2001). A non-probabilistic prospective and retrospective human reliability analysis method - application to railway system. Reliability Engineering and System Safety, 71 , pp. 1-13.

[35]Vanderhaegen, F., Crévits, I., Debernard, S. \& Millot, P. (1994), "Human-Machine Cooperation: Toward an Activity regulation assistance for different Air Traffic Control Levels", International Journal of Human Computer Interaction, 6 (1), 65-104.

[36] Klein GA, Calderwood R., (1991). Decision models: some lessons from the field, IEEE Transactions on Systems, Man and Cybernetics;21(5):1018-1026.

[37] Smith GF., (1989). Representational effects on the solving of an unstructured decision problem. IEEE Transactions on Systems, Man and Cybernetics 1989;19(5):1083 - 1090.

[38] Hoc JM., (1996) Supervision et contrôle de processus: la cognition en situation dynamique. Presse Universitaire de Grenoble, Collection Sciences \& Technologie de la connaissance.

[39] Klein G, Woods DD, Bradshaw JM, Hoffman RR, Feltovich PJ. (2004). Ten challenges for making automation a 'team player' in joint human-agent activity. IEEE Intelligent Systems;19(6):91-95. 